\title{
In vitro effects of lonidamine and 6-aminonicotinamide against Echinococcus granulosus sensu stricto and Echinococcus multilocularis
}

\author{
Qi Xin ${ }^{1}$, Miaomiao Yuan ${ }^{1,2}$, Huanping Li ${ }^{1}$, Xiaoxia Song ${ }^{1}$, Jun $\mathrm{Lu}^{1}$ and Tao Jing ${ }^{1 *}(\mathbb{C}$
}

\begin{abstract}
Echinococcosis is a zoonotic disease caused by cestode species of the genus Echinococcus, which demonstrates considerable medical and veterinary concerns. The development of novel drugs for echinococcosis treatment is urgently needed. In this study, we demonstrated that lonidamine (LND) and 6-aminonicotinamide (6-AN) exhibited considerable in vitro effects against both larval- and adult-stage of E. granulosus sensu stricto (s. s.) and E. multilocularis. The combination of $L N D$ and 6-AN exhibited a significantly higher activity than the single drug treatment. These results highlight the therapeutic potential of LND, 6-AN and the combination of LND and 6-AN for the treatment of echinococcosis.
\end{abstract}

\section{Introduction, methods and results}

Echinococcosis, acquired by the infection with the larval stage of the genus Echinococcus, is a severe and lifethreatening zoonotic disease. Cystic echinococcosis (CE) is caused by Echinococcus granulosus sensu lato (s. l.) and alveolar echinococcosis (AE) by Echinococcus multilocularis. The adult tapeworms inhabit in the intestine of the definitive hosts (mainly dogs for E. granulosus s. l. and foxes and dogs for E. multilocularis) and both diseases are acquired through the accidental ingestion of parasite eggs shed by definitive hosts. The parasites often result in organ dysfunction or even failure in humans and domestic livestock, causing severe public health problem and economic loss.

Albendazole (ABZ) is the currently used drug against echinococcosis, however, it only shows about $30 \%$ of cure rate against $\mathrm{CE}[1]$. As $\mathrm{ABZ}$ only has parasitostatic but

\footnotetext{
*Correspondence: jtao@lzu.edu.cn

${ }^{1}$ Institute of Pathogenic Biology, School of Basic Medical Sciences, Lanzhou University, Lanzhou, China

Full list of author information is available at the end of the article
}

not parasitocidal effect in $\mathrm{AE}$ patients, the patients must take the drug for a lifelong time to avoid recurrences and spread of metacestodes tissue [2], which unavoidably results in many adverse effects, such as nausea, hepatotoxicity and neutropenia. Therefore, it is necessary to identify novel potential chemotherapeutical treatment options for echinococcosis.

In E. multilocularis infection, metacestodes exhibit typical biological characteristics, such as progressive tumorlike growth and metastasize to other organs. Therefore, it is a useful and economical strategy to identify novel chemotherapeutics against Echinococcus by investigating the drugs inhibiting cancer cells proliferation [3]. A number of antitumor drugs, including 2-methoxyestradiol, imatinib, doxorubicin, cyclosporine, isoflavone genistein, rapamycin, bortezomib, tamoxifen, and 5-fluorouracil have been investigated for the treatment of echinococcosis. Lonidamine (LND) is a derivative of indazole-3-carboxylic acid, and exhibits effects against many cancer cell lines [4]. Moreover, this agent has gone through a multitude of clinical trials as a single agent or in combination 
with other anticancer chemotherapeutics (such as cisplatin, diazepam, and paclitaxel) [5]. 6-aminonicotinamide (6-AN), an analogue of nicotinamide, exhibits anticancer activity [6], and particularly, when used in combination with other anticancer agents, sensitizes cancer cells to those agents $[7,8]$. Therefore, we speculated that LND and 6-AN could exert effective activities against parasitic helminthes, including Echinococcus. In this study, we investigated the in vitro effects of LND, 6-AN and the combination of LND and 6-AN against E. granulosus sensu stricto (s. s.) and E. multilocularis.

Echinococcus granulosus s. s. protoscoleces were isolated aseptically from hydatid cysts in the liver of naturally infected sheep from a slaughter house in Xining, Qinghai, China. One hundred protoscoleces per well were cultured in $1 \mathrm{~mL}$ RPMI 1640 culture medium (supplemented with $12 \mathrm{mM}$ HEPES, $2 \mathrm{mM}$ glutamine, 100 $\mathrm{U} / \mathrm{mL}$ penicillin, and $100 \mu \mathrm{g} / \mathrm{mL}$ streptomycin) without phenol red. The drugs were added to the cultures at the following concentrations: $40 \mu \mathrm{M}$ nitazoxanide (NTZ), $40 \mu \mathrm{M}$ LND, $40 \mu \mathrm{M}$ 6-AN, $20 \mu \mathrm{M}$ LND $+20 \mu \mathrm{M}$ 6-AN and $40 \mu \mathrm{M} \mathrm{LND}+40 \mu \mathrm{M}$ 6-AN. Protoscoleces incubated in culture medium containing $0.2 \%$ dimethyl sulfoxide (DMSO) served as a control. Protoscoleces were observed microscopically every day and viability was evaluated by trypan blue exclusion test [9]. Each treatment was performed in duplicate and experiments were repeated twice. As positive control, NTZ killed 75\% protoscoleces after 4 days of treatment, and $100 \%$ after 5 days (Figure 1A). Comparatively, 6-AN killed 65\% and $76 \%$ protoscoleces, after 5 and 7 days of treatment, respectively, while LND killed $82 \%$ protoscoleces after 5 days and $100 \%$ protoscoleces after 6 days. The results demonstrated that LND exhibited higher efficacy than 6-AN, but slightly less than did NTZ. When $40 \mu \mathrm{M}$ LND and $40 \mu \mathrm{M} 6$-AN were applied in combination, the efficacy was markedly increased, with $86 \%$ of protoscoleces killed after 2 days of treatment and 100\% after 3 days. The combination of $20 \mu \mathrm{M}$ LND $+20 \mu \mathrm{M}$ 6-AN resulted in an eradication of $100 \%$ protoscoleces after 5 days of treatment. This effect was stronger than $40 \mu \mathrm{M}$ LND or $40 \mu \mathrm{M}$ 6-AN alone as well as NTZ (Figure 1A), and fully demonstrated the significant synergism of the combination. The morphological changes of protoscoleces after a 3-day treatment with LND, 6-AN or their combination further confirmed their efficacy (Figure 1B). Compared with control protoscoleces, most protoscoleces treated with LND or 6-AN exhibited distinct alterations, such as distortion and vesiculation, collapse of suckers, and decrease of calcareus corpuscles. Moreover, the combination of LND and 6-AN induced more severe damages than that did both drugs alone, with apparent destruction of parenchyma.

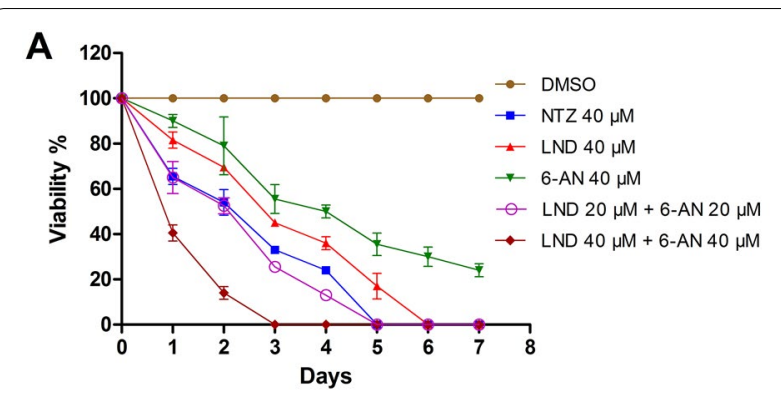

B

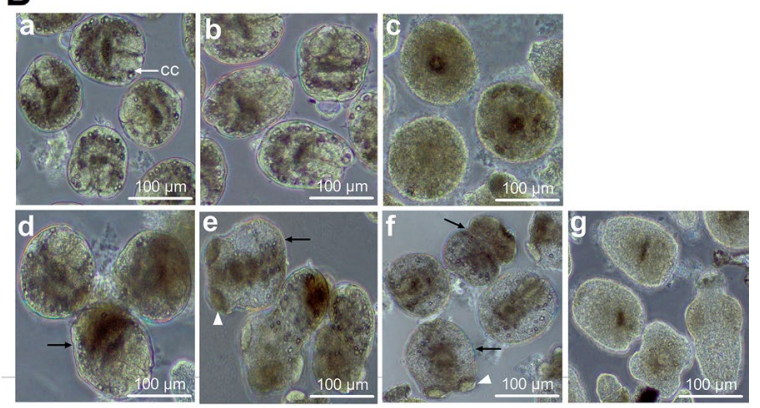

Figure 1 In vitro efficacy of the drugs against E. granulosus s. s. protoscoleces. A Survival of protoscoleces after treatment with LND, 6-AN, and their combination. B Morphologies of protoscoleces after 3 days of treatment with LND, 6-AN, and their combination. a Untreated protoscoleces. White arrow points towards calcareus corpuscles (cc); b Protoscoleces in culture medium containing 0.2\% DMSO; c, d, and e Protoscoleces incubated with $40 \mu \mathrm{M} \mathrm{NTZ,} 40 \mu \mathrm{M}$ 6-AN and $40 \mu M$ LND, respectively; f Protoscoleces incubated with $20 \mu \mathrm{M}$ LND $+20 \mu \mathrm{M}$ 6-AN. Note the distortion and vesiculation of protoscoleces (black arrow), the collapse of suckers (white arrowhead) and the decrease of calcareus corpuscles; $\mathbf{g}$ Protoscoleces incubated with $40 \mu \mathrm{M} L N D+40 \mu \mathrm{M} 6-\mathrm{AN}$.

To evaluate the activities of the drugs against E. multilocularis metacestodes (isolate Xinjing), the metacestodes were dissected from experimentally infected Mongolian gerbils (Meriones unguiculatus) and the in vitro culture of metacestodes was performed, as previously described [10]. The drug concentrations used were the same as above. After 36 and $120 \mathrm{~h}$ of incubation, culture supernatants were collected for measurement of $E$. multilocularis alkaline phosphatase (EmAP) activity, as previously described [11]. The experiments were performed in triplicate and repeated thrice. The results are presented as the mean \pm standard deviation and analyzed by One-way ANOVA using SPSS 19.0 software. After $36 \mathrm{~h}$ of treatment, LND and 6-AN both induced an increased release of EmAP activity in culture supernatants (Figure 2A), which indicated a drug-induced damage of vesicle cells from $E$. multilocularis metacestodes. The EmAP activities were strongly increased after $120 \mathrm{~h}$ of treatment, demonstrating the time-dependent effects of the drugs on the vesicles. Moreover, LND induced a 

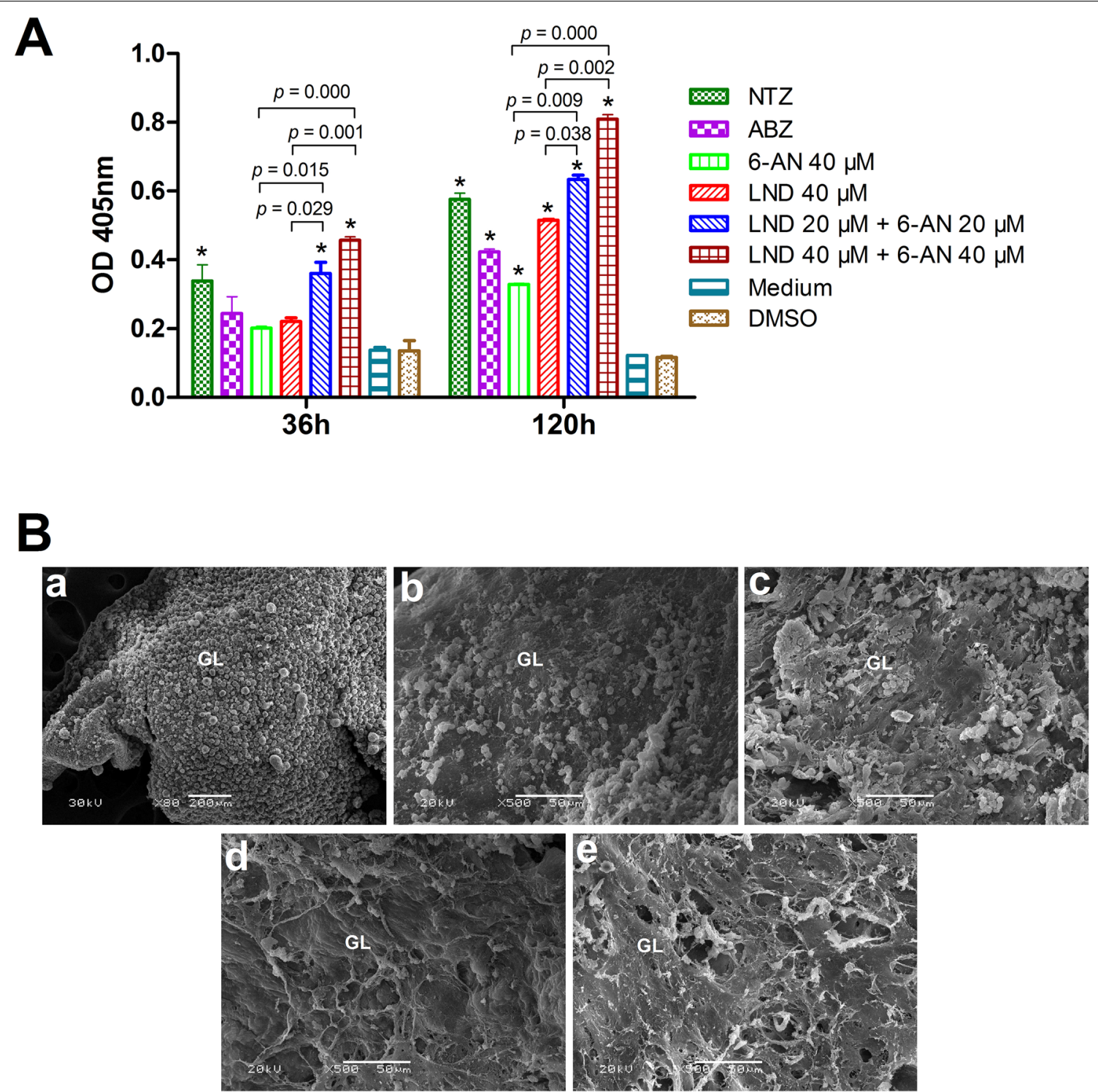

Figure 2 In vitro efficacy of the drugs against E. multilocularis metacestodes. A The release of alkaline phosphatase activity from $E$. multilocularis metacestodes (EmAP) after treatment with LND, 6-AN, and their combination. * $p<0.05$ vs. DMSO control. B Morphologies of metacestodes after 5 days of treatment with LND, 6-AN, and their combination. a Metacestodes in culture medium containing 0.2\% DMSO. Note

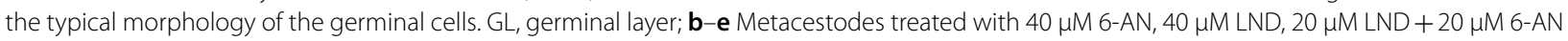
and $40 \mu \mathrm{M} L N D+40 \mu \mathrm{M} 6-\mathrm{AN}$, respectively.

significant increase in EmAP activity compared with ABZ treatment. There was no significant difference by comparison with NTZ treatment. For similar treatment period, $20 \mu \mathrm{M} \mathrm{LND}+20 \mu \mathrm{M} 6$-AN resulted in a higher release of EmAP than did $40 \mu \mathrm{M} L N D, 40 \mu \mathrm{M}$ 6-AN, and $40 \mu \mathrm{M}$ ABZ. In addition, $40 \mu \mathrm{M}$ LND $+40 \mu \mathrm{M} 6$ - AN induced a significant increase in EmAP activity than the other groups, including NTZ treatment. Such increase in EmAP activity coincided with the morphological alterations observed by scanning electron microscopy (SEM)
(Figure 2B). The metacestodes treated with 6-AN or LND exhibited noticeable damages, including the detachment of the germinal layer (GL) cells and the residual cellular materials in the GL. In comparison to 6-AN, LND treatment resulted in more stronger damages on metacestodes. Moreover, $20 \mu \mathrm{M} \mathrm{LND}+20 \mu \mathrm{M}$ 6-AN caused more severe damages than $40 \mu \mathrm{M}$ LND or $40 \mu \mathrm{M}$ 6 -AN. The most pronounced damage on metacestodes was observed after $40 \mu \mathrm{M} \mathrm{LND}+40 \mu \mathrm{M}$ 6-AN treatment, with the loss of the cellular integrity of the GL and 
complete disintegration of the major portion of the GL tissues.

Next, we evaluated the effects of LND, 6-AN and their combination against adult E. granulosus s. s.. Two beagle canines were orally infected with E. granulosus s. s. protoscoleces from hydatid cysts from naturally infected sheep. Thirty-three days after infection, adult E. granulosus s. s. were collected from the small intestine of euthanized dogs and 35 tapeworms were placed into $3 \mathrm{~mL}$ of RPMI 1640 culture medium without phenol red. Animal procedures and management were carried out in accordance with the protocols (2018-03-002) approved by the Institutional Animal Caring and Using Committee of Lanzhou University. The drugs were added to the cultures at the following concentrations: $200 \mu \mathrm{M}$ praziquantel (PZQ), $200 \mu \mathrm{M}$ LND, $200 \mu \mathrm{M}$ 6-AN, $100 \mu \mathrm{M}$ LND $+100 \mu \mathrm{M}$ 6-AN and $200 \mu \mathrm{M}$ LND $+200 \mu \mathrm{M}$ 6-AN. After incubation for $4 \mathrm{~h}$ at $37{ }^{\circ} \mathrm{C}$ with $5 \% \mathrm{CO}_{2}$, culture supernatants were collected for measurement of E. granulosus s. s. alkaline phosphatase (EgAP) activity. Significant increases in $\mathrm{EgAP}$ activity from culture supernatants were observed after treatment with LND, 6-AN, $100 \mu \mathrm{M}$ LND $+100 \mu \mathrm{M}$ 6-AN, $200 \mu \mathrm{M}$ LND + $200 \mu \mathrm{M}$ 6-AN and $\mathrm{PZQ}$, in comparison to DMSO control (Figure 3A). LND induced a significant increase in EgAP activity compared with 6-AN, while had no difference with PZQ. $100 \mu \mathrm{M} \mathrm{LND}+100 \mu \mathrm{M}$ 6-AN resulted in more significant increase in EgAP activity than did $200 \mu \mathrm{M}$ LND or $200 \mu \mathrm{M}$ 6-AN. The most significant increase in EgAP activity was induced by $200 \mu \mathrm{M}$ LND $+200 \mu \mathrm{M}$ 6-AN, in comparison to LND or 6-AN alone, and even PZQ. The morphological alterations of adult E. granulosus $s$. $s$. after treatment were consistent with the EgAP activity assay. For optical microscopy (Figure 3B), tapeworms in DMSO control maintained a typical appearance: an integrated scolex composed of a rostellum, intact hooks and four concave suckers with a distinct neck and three proglottids. In comparison, 6-AN and LND treated tapeworms exhibited extensive alterations, including the dimming and distortion of body segments, partial loss of hooks on the rostellum, and contraction and distortion of the suckers. $100 \mu \mathrm{M}$ LND $+100 \mu \mathrm{M}$ 6-AN and $200 \mu \mathrm{M}$ $\mathrm{LND}+200 \mu \mathrm{M} 6$-AN both resulted in more pronounced changes than those induced both drugs alone. Noticeably, the morphological alterations caused by PZQ were quite different from those caused by LND and 6-AN, showing more starker dimming, swelling and shortening of the body segments (especially the first and second proglottid), destruction of the tegument, and severe distortion of the rostellum and suckers. Transmission electron microscopy (TEM) (Figure 3C) showed that the tapeworms from DMSO control had the intact and typical structure: the smooth and conical microtriches covered the intact tegument, round or oval microfilaments at the base of the microtriches, multiple mitochondrion presented within the distal cytoplasm and the basement membrane located at the bottom of the cytoplasm, the subcutaneous layer contained circular and longitudinal muscle bundles and perikarya. In comparison, 6-AN treated tapeworms exhibited partial shedding of microtriches and cellular destruction of perikarya. LND resulted in a marked reduction in the size and number of microtriches and destruction of the substantial portions of subcutaneous layer. $100 \mu \mathrm{M} \mathrm{LND}+100 \mu \mathrm{M}$ 6-AN resulted in more severe damages than did $200 \mu \mathrm{M}$ LND or $200 \mu \mathrm{M}$ 6-AN, with noticeable truncated and reduced microtriches and the residual perikarya which presented electrondense and swollen mitochondrion. Moreover, $200 \mu \mathrm{M}$ $\mathrm{LND}+200 \mu \mathrm{M} 6$-AN resulted in the most severe damages, exhibiting severely damaged perikarya and amorphous organellar debris, and occurrence of lipid droplets. As observed by optical microscopy, PZQ resulted in different alterations from those induced by 6-AN and LND, with the outer covering microtriches completely disrupted.

\section{Discussion}

This study investigates for the first time the in vitro effect of the anti-cancer drugs LND, 6-AN and the combination of LND and 6-AN against E. granulosus s. s. and E. multilocularis. LND and 6-AN both exhibited considerable efficacy against $E$. granulosus s. s. protoscoleces and E. multilocularis in a time dependent manner, particularly the efficacy of LND was more efficacious than that of $\mathrm{ABZ}$, the current licensed drug for human echinococcosis. It is not surprising that LND has profound efficacy against Echinococcus, as it has exhibited a spectrum of antineoplastic activities [4,5] and efficacy against several protozoan parasites, including Leishmania mexicana [12], Trypanosoma cruzi and Trypanosoma brucei [13]. However, the mechanism of action of LND against Echinococcus is not clear. Some studies reported that LND may interfere with cellular energy by inhibiting mitochondrial hexokinases [14] and mitochondrial electron transport [15] in cancer cells. Furthermore, the mechanism of the inhibition of L. mexicana by LND is through inhibition of energy metabolism [12] and for T. brucei, inhibition of hexokinase activity [16]. The previous studies revealed that although E. granulosus s. s. and E. multilocularis both have aerobic and anaerobic respiratory systems, the energy and intermediates production were mainly dependent on glycolysis $[17,18]$. Thus, the interference with glycolysis by inhibiting hexokinase might be the mechanism of LND against Echinococcus. 


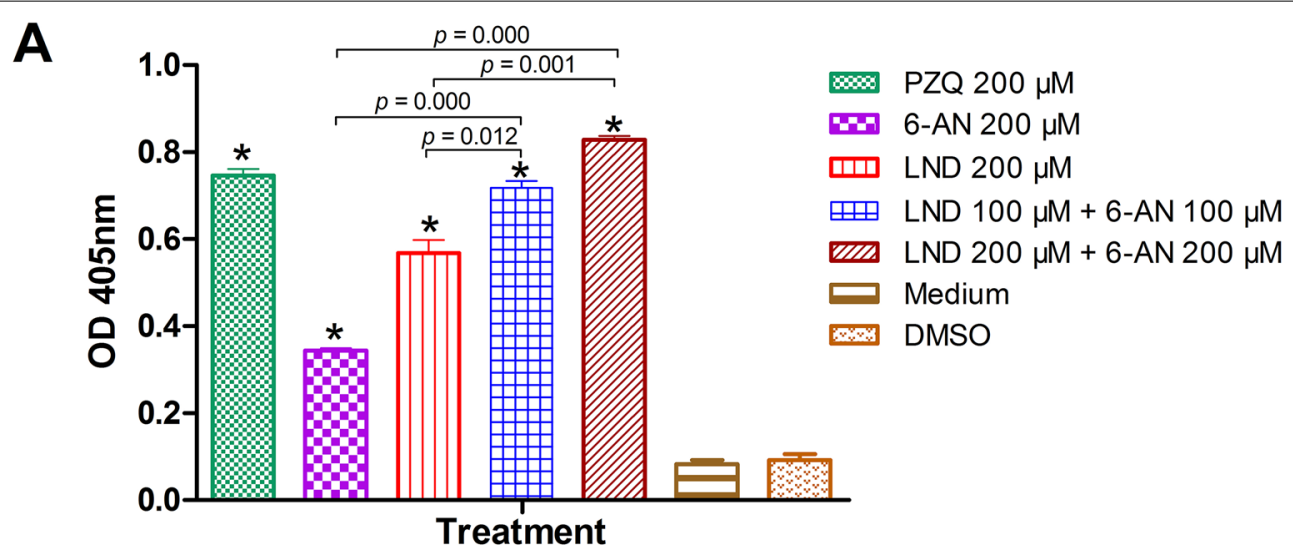

B

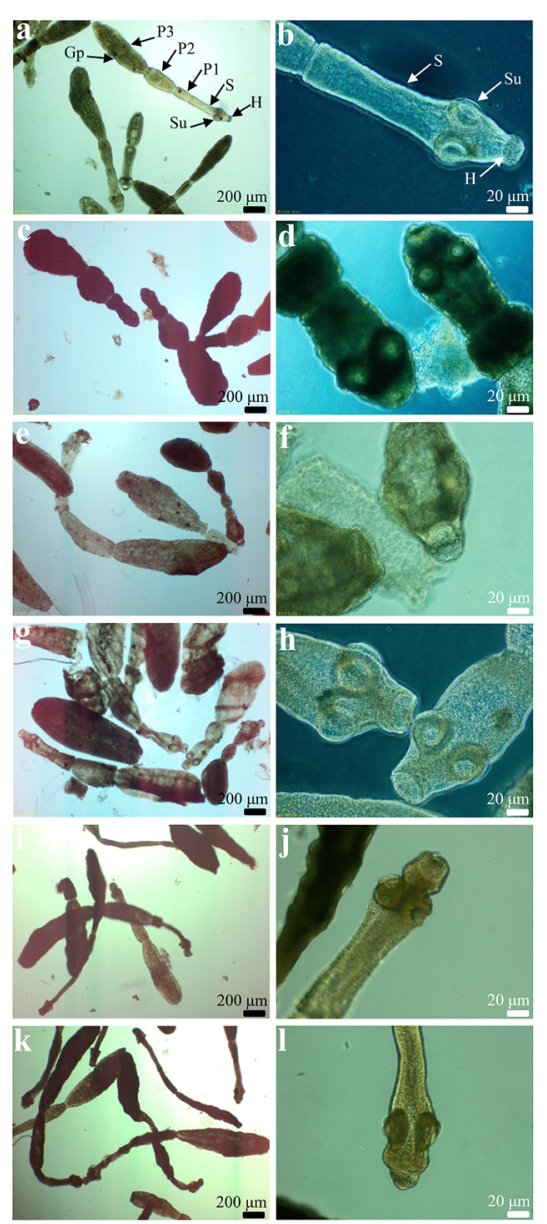

\section{C}
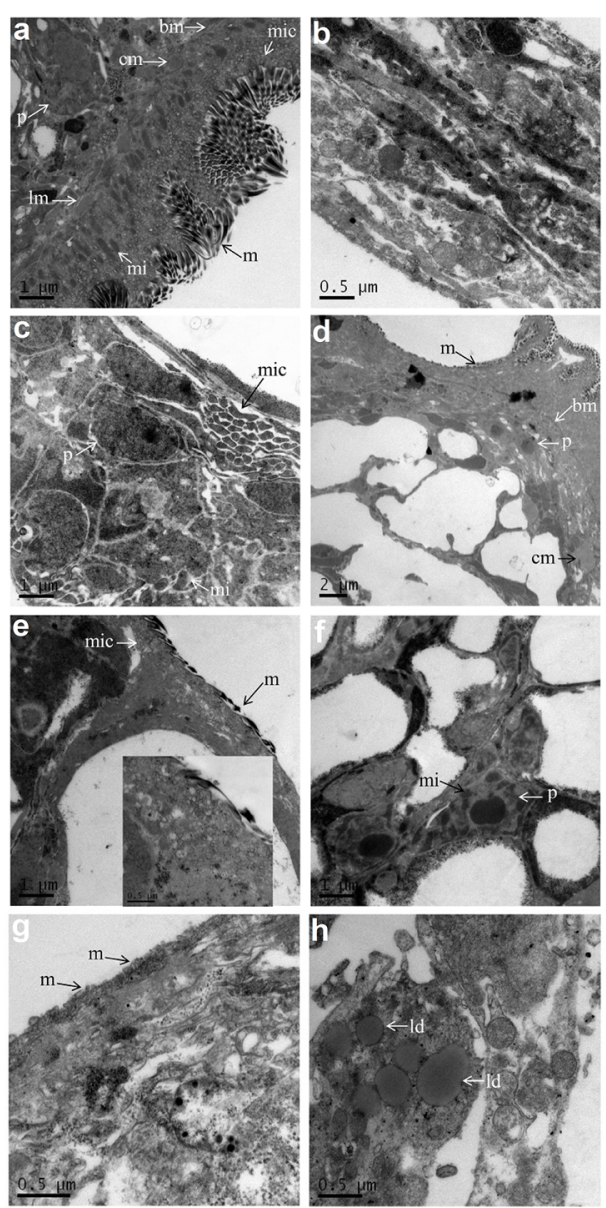

Figure 3 In vitro efficacy of the drugs against adult E. granulosus s. s. A The release of alkaline phosphatase from adult E. granulosus s. S. (EgAP) after treatment with LND, 6-AN, and their combination. ${ }^{*} p<0.05$ vs. DMSO control. B Morphologies of adult E. granulosus s. s. after treatment with LND, 6-AN, and their combination. $\mathbf{a}$, b Tapeworms incubated in culture medium containing $0.2 \%$ DMSO. Note the concave suckers (Su) on the scolex (S), the intact first proglottid (P1), second proglottid (P2) and terminal proglottid (P3) with genital pore (Gp) as well as the complete

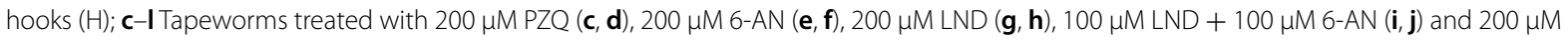
LND + $200 \mu \mathrm{M}$ 6-AN (k, I), respectively; C Ultrastructures of adult E. granulosus s. s. after $4 \mathrm{~h}$ of treatment with LND, 6-AN, and their combination. a Tapeworms incubated in culture medium containing 0.2\% DMSO. Note the normal and distinct morphology ( $\mathrm{m}$, microtriches; mi, mitochondria; mic, microfilament; $\mathrm{cm}$, circular muscle bundle; Im, longitudinal muscle bundle; bm, basement membrane; $\mathrm{p}$, perikarya) and the large number and the length of microtriches; b-h Tapeworms treated with $200 \mu \mathrm{M}$ PZQ (b), $200 \mu \mathrm{M}$ 6-AN (c), $200 \mu \mathrm{M}$ LND (d), $100 \mu \mathrm{M}$ LND + $100 \mu \mathrm{M}$ 6-AN (e, f) and $200 \mu \mathrm{M}$ LND + $200 \mu \mathrm{M}$ 6-AN (g, g), respectively. Id: lipid droplet. 
LND produced a greater anti-Echinococcus effect than that observed with 6-AN. LND killed 100\% of E. granulosus s. s. protoscoleces after 6 days of treatment. In contrast, 6-AN treatment killed 76\% protoscoleces after 7 days (Figure $1 \mathrm{~A})$. In addition, on E. multilocularis metacestodes, LND resulted in $0.085 \pm 0.002$ and $0.400 \pm 0.001$ OD405 $\mathrm{nm}$ increase at $36 \mathrm{~h}$ and $120 \mathrm{~h}$ of treatment respectively compared with the DMSO group, higher than that with 6 -AN $(0.066 \pm 0.002 ; 0.212 \pm 0.001)$ (Figure $2 \mathrm{~A})$. Likewise, LND produced a higher increase in OD405 nm $(0.477 \pm 0.014)$ than did 6-AN $(0.252 \pm 0.004)$ on adult $E$. granulosus s. s. (Figure 3A). The morphological and ultrastructural alterations confirmed these effects (Figures 1B, $2 \mathrm{~B}$ and $3 \mathrm{~B})$. As we known, in the past years, 6-AN, which is believed to be a competitive inhibitor of glucose-6-phosphate dehydrogenase (G6PD) [19, 20], could inhibit G6PD to catalyse the conversion of glucose-6-phosphate (G6P) to 6 -phosphoglucono- $\delta$-lactone (6PG $\delta \mathrm{L}$ ), the first step of the pentose phosphate pathway (PPP) and has been used for chemotherapy of various tumors [6]. Therefore, the possible mechanism of 6-AN against Echinococcus might be through inhibiting the PPP, an alternate metabolic pathway involved in energy production and can generate NADPH and important metabolic intermediates (pentose-5-phosphate) for synthesis of macromolecules [21, 22].

In addition to the efficacy against the larval stage of E. granulosus s. s. and E. multilocularis, LND and 6-AN also exhibit significant effects on the adult stage of $E$. granulosus s. s. (Figure 3). As we known, the adult tapeworms inhabit in the intestine of the definitive hosts at a relative anaerobic environment and depend on glycolysis for energy and intermediates production [23], and thus, the adult tapeworms are sensitive to LND and 6-AN. Furthermore, as observed by optical microscopy and TEM, the morphological and ultrastructural alterations induced by LND and 6-AN were different from the changes in PZQ-treated parasite (Figure 3B). LND and 6-AN mainly induced destruction of substantial portions of subcutaneous layer but microtriches still remained in the tegument, while PZQ completely disrupted the outer covering microtriches and the integrity of tegument. The same alterations by PZQ have also been reported by Conder et al. [24]. The different changes probably correspond to the different mode of action of the drugs. PZQ treatment results in osmotic lytic alterations of tegument, the loss of organic material from tapeworm and thus leads to the death of parasites $[24,25]$. In contrast, LND and 6-AN may interfere with glucose and respiratory metabolism, respectively.

Our results demonstrated that the combination of LND and 6-AN had stronger effect than that did both drugs alone. Moreover, the combination exhibited not only the best efficacy, but also the faster action. At the same final concentration, the combination killed $100 \%$ protoscoleces after 5 days of treatment, to be compared with $100 \%$ protoscoleces killed after 6 days of treatment with LND and 76\% protoscoleces killed after 7 days treatment with 6-AN (Figure 1). This characteristic was confirmed by EmAP activity assay (Figure 2A), morphological and ultrastructural alterations. We suppose the enhanced effect by the combination might be due to the complementary disruption of biochemical pathways by LND and 6-AN. As a competitive G6PD inhibitor, 6-AN could suppress PPP reduce the cellular energy, and impair DNA synthesis and repair. Both LND and 6-AN induce depletion of metabolic energy, yet they target different sites in energy generation pathways. Their use in combination might enhance energy depletion, and thereby yield significant effect against Echinococcus. Therefore, it suggests that the inhibition of both pathways of energy production may achieve synergetic effect against Echinococcus. As we known, toxicity, weak efficacy, and especially rapidly increasing drug-resistance hinder the effectiveness of chemotherapy to numerous diseases including echinococcosis. Therefore, the combination of drugs is one of the solutions to the above problems, which has many advantages including the enhancement of efficacy and delay of drug resistance [26].

In conclusion, in the present study, we demonstrate that LND and 6-AN exhibit considerable effects against both larval- and adult-stage of E. granulosus s. s. and $E$. multilocularis. Furthermore, the combined treatments with LND and 6-AN exhibit stronger effects than that did both drugs alone. The present results demonstrate the therapeutic potential of LND, 6-AN, and particularly their combination against E. granulosus s. $s$. and E. multilocularis. Further studies will be performed by investigating the in vivo efficacy of LND, 6-AN and their combination against Echinococcus spp. as well as the mechanism of action.

\section{Acknowledgements \\ This research was supported by NSFC (Grant No. 81171632), the Fundamental Research Funds for the Central Universities (Protocol code Izujbky-2018-90). \\ Authors' contributions \\ TJ conceived this project, designed the experiments and reviewed the manuscript. QX and MY designed and performed the experiment, analyzed and interpreted the data and drafted the manuscript. $\mathrm{HL}, \mathrm{XS}$ and $J \mathrm{~L}$ partici- pated in performing the experiment. All authors read and approved the final manuscript.}

\section{Competing interests}

The authors declare that they have no competing interests.

\section{Author details}

${ }^{1}$ Institute of Pathogenic Biology, School of Basic Medical Sciences, Lanzhou University, Lanzhou, China. ${ }^{2}$ The Eighth Affiliated Hospital, Sun Yat-sen University, Shenzhen, China.

Received: 10 September 2019 Accepted: 22 January 2020 Published online: 26 February 2020 


\section{References}

1. Vuitton DA (2009) Benzimidazoles for the treatment of cystic and alveolar echinococcosis: what is the consensus? Expert Rev Anti Infect Ther 7:145-149

2. Reuter S, Buck A, Manfras B, Kratzer W, Seitz HM, Darge K, Reske SN, Kern P (2004) Structured treatment interruption in patients with alveolar echinococcosis. Hepatology 39:509-517

3. Hemphill A, Müller J (2009) Alveolar and cystic echinococcosis: towards novel chemotherapeutical treatment options. J Helminthol 83:99-111

4. Del Bufalo D, Biroccio A, Soddu S, Laudonio N, D'Angelo C, Sacchi A, Zupi G (1996) Lonidamine induces apoptosis in drug-resistant cells independently of the p53 gene. J Clin Invest 98:1165-1173

5. Pelicano H, Martin DS, Xu RH, Huang P (2006) Glycolysis inhibition for anticancer treatment. Oncogene 25:4633-4646

6. Gupte SA (2008) Glucose-6-phosphate dehydrogenase: a novel therapeutic target in cardiovascular diseases. Curr Opin Investig Drugs 9:993-1000

7. Budihardjo II, Walker DL, Svingen PA, Buckwalter CA, Desnoyers S, Eckdahl S, Shah GM, Poirier GG, Reid JM, Ames MM, Kaufmann SH (1998) 6-Aminonicotinamide sensitizes human tumor cell lines to cisplatin. Clin Cancer Res 4:117-130

8. Varshney R, Gupta S, Dwarakanath BS (2004) Radiosensitization of murine ehrlich ascites tumor by a combination of 2-deoxy-D-glucose and 6-aminonicotinamide. Technol Cancer Res Treat 3:659-663

9. Walker M, Rossignol JF, Torgerson P, Hemphill A (2004) In vitro effects of nitazoxanide on Echinococcus granulosus protoscoleces and metacestodes. J Antimicrob Chemother 54:609-616

10. Yuan M, Song X, Lv W, Xin Q, Wang L, Gao Q, Zhang G, Liao W, Lian S, Jing $T$ (2019) Effect of anacardic acid against echinococcosis through inhibition of VEGF-induced angiogenesis. Vet Res 50:3

11. Stettler M, Siles-Lucas M, Sarciron E, Lawton P, Gottstein B, Hemphill A (2001) Echinococcus multilocularis alkaline phosphatase as a marker for metacestode damage induced by in vitro drug treatment with albendazole sulfoxide and albendazole sulfone. Antimicrob Agents Chemother 45:2256-2262

12. Turrens JF, Cazzulo JJ (1987) Inhibition of growth and respiration of Leishmania mexicana by the antitumor agent lonidamine. Comp Biochem Physiol C 88:193-196

13. Turrens JF (1986) Inhibitory action of the antitumor agent lonidamine on mitochondrial respiration of Trypanosoma cruzi and T. brucei. Mol Biochem Parasitol 20:237-241

14. Paggi MG, Fanciulli M, Perrotti N, Floridi A, Zeuli M, Silvestrini B, Caputo A (1988) The role of mitochondrial hexokinase in neoplastic phenotype and its sensitivity to lonidamine. Ann N Y Acad Sci 551:358-360
15. Floridi A, Lehninger AL (1983) Action of the antitumor and antispermatogenic agent lonidamine on electron transport in Ehrlich ascites tumor mitochondria. Arch Biochem Biophys 226:73-83

16. Chambers JW, Fowler ML, Morris MT, Morris JC (2008) The anti-trypanosomal agent lonidamine inhibits Trypanosoma brucei hexokinase 1. Mol Biochem Parasitol 158:202-207

17. Agosin M (1957) Studies on the metabolism of Echinococcus granulosus. II. Some observations on the carbohydrate metabolism of hydatid cyst scolices. Exp Parasitol 6:586-593

18. Mcmanus DP, Smyth JD (1978) Differences in the chemical composition and carbohydrate metabolism of Echinococcus granulosus (horse and sheep strains) and E. multilocularis. Parasitology 77:103-109

19. Köhler E, Barrach H, Neubert D (1970) Inhibition of NADP dependent oxidoreductases by the 6-aminonicotinamide analogue of NADP. FEBS Lett 6:225-228

20. Lange K, Proft ER (1970) Inhibition of the 6-phosphogluconate dehydrogenase in the rat kidney by 6-aminonicotinamide. Naunyn Schmiedebergs Arch Pharmakol 267:177-180

21. Keniry MA, Hollander C, Benz CC (1989) The effect of gossypol and 6-aminonicotinamide on tumor cell metabolism: a 31P-magneticresonance spectroscopic study. Biochem Biophys Res Commun 164:947-953

22. Sharma RK, Hanssum H, Jain V (1996) In vivo NMR spectroscopic studies on the bioenergetic changes induced by metabolic modulators in Ehrlich ascites tumour cells. Indian J Biochem Biophys 33:122-130

23. Smyth JD, Mcmanus DP (1989) The physiology and biochemistry of cestodes. J Parasitol 77:271

24. Conder GA, Marchiondo AA, Andersen FL (1981) Effect of praziquantel on adult Echinococcus granulosus in vitro: scanning electron microscopy. Z Parasitenkd 66:191-199

25. Becker B, Mehlhorn H, Andrews P, Thomas H (1980) Scanning and transmission electron microscope studies on the efficacy of praziquantel on Hymenolepis nana (Cestoda) in vitro. Z Parasitenkd 61:121-133

26. Cottarel G, Wierzbowski J (2007) Combination drugs, an emerging option for antibacterial therapy. Trends Biotechnol 25:547-555

\section{Publisher's Note}

Springer Nature remains neutral with regard to jurisdictional claims in published maps and institutional affiliations.
Ready to submit your research? Choose BMC and benefit from:

- fast, convenient online submission

- thorough peer review by experienced researchers in your field

- rapid publication on acceptance

- support for research data, including large and complex data types

- gold Open Access which fosters wider collaboration and increased citations

- maximum visibility for your research: over 100M website views per year

At $\mathrm{BMC}$, research is always in progress.

Learn more biomedcentral.com/submissions 